\title{
Recent developments on bridge flutter stability estimation accounting for errors in the aeroelastic loading
}

\author{
L. Caracoglia \\ Department of Civil and Environmental Engineering, \\ Northeastern University, Boston, MA, USA
}

\begin{abstract}
State-space stochastic modeling (Itô-type) is employed in this study to numerically investigate the relevance of modeling errors in the aeroelastic loading on the flutter stability of a long-span bridge. The main features of the numerical model are: $(i)$ linear time-domain representation of aeroelastic load based on indicial-function formulation, perturbed by a suitable "parametric error"; (ii) turbulence-induced buffeting load still retained in the dynamic equations; (iii) linear bridge response restricted to fundamental bending and torsional mode of the moving deck and cables. The flutter velocity is determined by using the definition of second- and third-moment stability of the random dynamic vector. A simplified bridge model is employed to investigate the feasibility of the proposed approach. Two numerical algorithms for the solution of stochastic stability are implemented. The comparison of the results is based on statistical properties of the random state vector at incipient flutter (initial time), which includes the "parametric error" variable.

Keywords: long-span bridges, flutter, modeling errors, stochastic calculus, moment stability.
\end{abstract}

\section{Introduction}

Long-span bridges are large-period and low-damping complex structures, affected by wind hazards. Accurate assessment of flutter velocity is of relevance to the bridge designer, since this phenomenon may lead to structural collapse. Recent studies by the author aim at the development of numerical methods for 
the simulation of wind-induced response of a bridge, in which random "error" propagation in the dynamic system of equations is incorporated. This paper discusses recent results of a research investigation on the estimation of critical flutter velocity, influenced by experimental or modeling errors in the aeroelastic loading.

An analytically-based and numerically-implemented model, based on stochastic calculus, has been recently proposed to assess the coupled-flutter stability threshold of a bridge [1,2]. The presence of "errors" was indirectly included through parametric perturbation to selected variables. The proposed approach is distinct from other methods, developed in the recent past [3-6], for example employing stochastic calculus to analyze the effects of turbulence on flutter velocity. While in previous models a perturbation of the mean wind speed through a random "white noise" was used to simulate turbulence effects on flutter, the actual buffeting loading is retained in the approach proposed herein. Furthermore, even though nonlinear stochastic dynamics has been recently used for post-critical flutter investigation [7], few studies have considered the quantification of random error effects, when this error is directly "embedded" in the unsteady self-excited forces acting on the deck. In particular, the random perturbation is introduced in the parameters describing the "indicial functions" [8] of this loading.

The solution to the stochastic dynamic problem is based on an augmented state-space system. Stability is assessed in terms of mean squares and third-order statistical moments [9]. The use of two techniques for the solution of the differential equations is presented: $(i)$ a "Direct Euler - Monte Carlo" time marching algorithm [10], developed by the author to numerically calculate the statistical moments of the generalized modal coordinates of the deck modes; (ii) numerical estimation of moment Lyapunov exponents for stochastic stability [9]. A simulation example, modeling a realistic bridge structure, is discussed.

\section{Dynamic modeling of the bridge response: background}

A short description of the model for wind-induced analysis before the inclusion of random perturbation is discussed. The model is based on the "multi-mode approach" (e.g., $[11,12])$ for aeroelastic and aerodynamic response simulation. Small vibration amplitudes are postulated with respect to an initial equilibrium configuration of the deck under mean wind loads and an initial "attack angle" $\alpha_{0}$. The mean cross-flow speed $U$ acts orthogonally to the deck axis (Figure 1), since this direction coincides with the most unfavorable loading case.

The dynamic response is restricted to vertical $h$ displacements and angular rotation $\alpha$ of the generic deck cross section (Figure 1). Effects of the lateral motion component are ignored. Response is represented by modal superposition as a function of time $t$, longitudinal deck coordinate $x$ and deck width $B$ as [11]

$$
h(x, t)=\sum_{g} \xi_{g}(t) B h_{g}(x), \quad \alpha(x, t)=\sum_{g} \xi_{g}(t) \alpha_{g}(x)
$$


A reduced-order model is constructed from the modal expansion in eqn (1). The dynamic response is restricted to two modes, mainly responsible for coupled flutter: the fundamental flexural mode $(j)$ with $h_{j}(x) \neq 0$ in eqn (1), and the fundamental torsional mode $(k)$ with $\alpha_{k}(x) \neq 0$.

Lift forces $\left(L_{A E}, L_{b}\right)$ and torsional moments $\left(M_{A E}, M_{b}\right)$ in Figure 1 are included in the formulation by means of standard "strip theory" [11], whereas influence of drag forces $\left(D_{A E}\right.$ or $\left.D_{b}\right)$ is neglected.

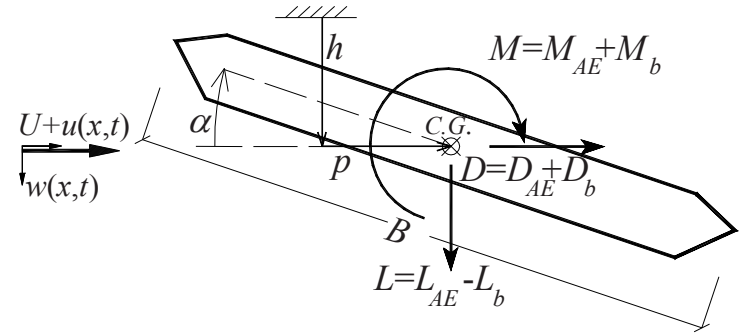

Figure 1: Aeroelastic and aerodynamic loads, degrees of freedom of the generic deck section - positive loading and displacement direction is shown.

Each generalized dynamic equation of mode $j$ and $k$ incorporates a linear unsteady fluid-structure interaction model, based on indicial functions (e.g., [8]). An indicial function (IF) models the unsteady temporal evolution of a selfinduced dimensionless force component due to a "unit-step" change in configuration of the deck at $s=0$, with $s=t U / B$ being a dimensionless time. The change of configuration is related either to angular rotation or vertical velocity. Each IF is referenced to the corresponding value of the static force coefficient per unit length.

For example, the IF of the torsional moment $M_{A E}$ due to a unit-step variation in the angle of attack $\alpha$ describes the instantaneous evolution of the torque with respect to $C_{M}{ }^{*}=d C_{M} / d \alpha$ evaluated at $\alpha_{0}$, with $C_{M}$ being the static torque coefficient [8]. This function is

$$
\Phi_{\alpha \alpha}(s)=1-\sum_{i=1}^{m_{\alpha \alpha}} c_{i, \alpha \alpha} \exp \left(-d_{i, \alpha \alpha} s\right) .
$$

In eqn (2) the subscript " $\alpha \alpha$ " identifies coupling between aeroelastic torque and torsional motion; $c_{i, \alpha \alpha}$ and $d_{i, \alpha \alpha}$, are linear parameters obtained from the flutter derivatives (FDs), which are measured experimentally in wind tunnel [13]. The "link" between experimental values of the FDs and eqn (2) is based on nonlinear regression of $c_{i, \alpha \alpha}, d_{i, \alpha \alpha}$, with pre-selected model order $m_{\alpha \alpha}$ [14]. Expressions similar to eqn (2) can be postulated for the IF associated with $M_{A E}$ and a variation in $d h / d t\left(\Phi_{a h}(s)\right.$ with parameters $C_{M}{ }^{*}, c_{i, \alpha h}, d_{i, \alpha h}$ and order $\left.m_{\alpha h}\right)$, and for the lift-force IFs, dependent on either a step-variation of $\alpha, \Phi_{h \alpha}(s)$ or vertical velocity, $\Phi_{h h}(s)$. In the latter two cases the IFs depend on $C_{L}{ }^{*}=d C_{L} / d \alpha$ at $\alpha_{0}$, with $C_{L}$ being the static lift coefficient. 
The combination of eqns (1) and (2), after modal superposition, leads to a pair of dynamic equations (for $j$ and $k$ ). As an example, the equation of mode $k$ is

$$
\xi_{k}^{\prime \prime}=\left\{\begin{array}{l}
{\left[-K_{k}^{2}+\Phi_{h \alpha, 0} \Lambda_{k k, h \alpha}+\Phi_{\alpha \alpha, 0} \Lambda_{k k, \alpha \alpha}\right] \xi_{k}(s)+\left[\Phi_{h \alpha, 0} \Lambda_{k j, h \alpha}+\Phi_{\alpha \alpha, 0} \Lambda_{k j, \alpha \alpha}\right] \xi_{j}(s)} \\
+\left[\Phi_{h h, 0} \Lambda_{k k, h h}+\Phi_{\alpha h, 0} \Lambda_{k k, \alpha h}-2 \zeta_{k} K_{k}\right] \xi_{k}^{\prime}(s)+\left[\Phi_{h h, 0} \Lambda_{k j, h h}+\Phi_{\alpha h, 0} \Lambda_{k j, \alpha h}\right] \xi_{j}^{\prime}(s) \\
+\sum_{g=j, k} \sum_{r=h, \alpha}\left[\Lambda_{k g, p h} \sum_{i=1}^{m_{r h}} v_{a e, g, r h, i}(s)\right]+\sum_{g=j, k} \sum_{r=h, \alpha}\left[\Lambda_{k g, p \alpha} \sum_{i=1}^{m_{r \alpha}} \mu_{a e, g, r \alpha, i}(s)\right]+F_{b, k}(s)
\end{array}\right\} .
$$

In eqn (3) the prime super-script symbol denotes derivation with respect to $S$, $\Phi_{h h, 0}=\Phi_{h h}(s=0), \quad \Phi_{h \alpha, 0}=\Phi_{h \alpha}(s=0), \quad \Phi_{\alpha h, 0}=\Phi_{\alpha h}(s=0) \quad$ and $\quad \Phi_{\alpha \alpha, 0}=\Phi_{\alpha \alpha}(s=0) . \quad$ Also, structural vibration in eqn (3) is expressed in terms of reduced frequency $K_{k}=\omega_{k} B / U$ (with $k$-th mode circular frequency $\omega_{k}$ ) and damping ratio $\zeta_{k}$. A dimensionless group $\Lambda_{j k, r q}=G_{r_{j} q_{k}} \Gamma_{r}\left(0.5 \rho B^{4} \ell / I_{k}\right)$ is used with $r, q=h, \alpha$ and: $I_{k}$, generalized modal inertia; $\ell$, bridge length; $\Gamma_{h}=-C_{L}^{*}, \Gamma_{\alpha}=C_{M}^{*}$. The "modal integrals" $G_{r_{j} q_{k}}$, are defined as in [11], e.g., $G_{h_{j} \alpha_{k}}=\int_{0}^{\ell} h_{j}(x) \alpha_{k}(x) d x / \ell$.

The modal aeroelastic load in eqn (3) is represented by $v_{a e, g, r q, i}$ and $\mu_{a e, g, r q, i}$, a series of $i=1, \ldots, m_{r q}$ "aeroelastic states" of mode $g=j$ or $k$ with components $r, q=h, \alpha[4]$. These states depend on the generalized coordinates and the IFs. Other state-space equations are needed [15] to describe the temporal evolution of $v_{a e, g, r q, i}$ and $\mu_{a e, g, r q, i}$ for the generic mode $g$. These can be derived [15] as: $v_{a e, r q, g, i}^{\prime}=d_{i, r q} c_{i, r q} \xi_{g}^{\prime}-d_{i, r q} v_{a e, r q, g, i}, \mu_{a e, r q, g, i}^{\prime}=d_{i, r q} c_{i, r q} \xi_{g}-d_{i, r q} \mu_{a e, r q, g, i}$.

The modal buffeting load is described in eqn (3) by $F_{b, k}(s)$. This term is expressed as a linear function of zero-mean stationary turbulence components $\hat{u}(s)=u(s) / U$ (horizontal) and $\hat{w}(s)=w(s) / U$ (vertical). Contrary to multimode approach (e.g., [11]) or other standard time-domain aerodynamic modeling (e.g., [15]), the loading is simulated as a correlated generalized force $\left(L_{b}\right.$ and $M_{b}$ in Figure 1) in terms of time $s$. The actual loss of load correlation is indirectly accounted for through dimensionless "modal span-wise correlation lengths" [1]. The correlation length of mode $j$ and generalized lift $\left(\bar{L}_{\psi_{h j}}\right)$ and the one of mode $k$ and torque $\left(\bar{L}_{\psi_{\alpha k}}\right)$ can be related to $\hat{w}(s)$ by least squares [1] as

$$
\begin{gathered}
{\left[\left(C_{L}^{*}+C_{D}\right) \bar{L}_{\psi_{h j}}\right]^{2}=\ell^{-2} \iint\left(C_{L}^{*}+C_{D}\right)^{2} h_{j}\left(x_{1}\right) h_{j}\left(x_{2}\right) \mathrm{e}^{\left(-c_{w j}\left|x_{1}-x_{2}\right| \ell \ell\right.} d x_{1} d x_{2},} \\
{\left[C_{M}^{*} \bar{L}_{\psi_{\alpha k}}\right]^{2}=\ell^{-2} \iint C_{M}^{*}{ }^{2} \alpha_{k}\left(x_{1}\right) \alpha_{k}\left(x_{2}\right) \mathrm{e}^{\left(-c_{w k}\left|x_{1}-x_{2}\right| / \ell\right)} d x_{1} d x_{2} .}
\end{gathered}
$$

Equations (4) and (5) are dimensionless with double integration carried out over $\ell ; c_{w j}$ and $c_{w k}$ are two constants (e.g., [11]); while $C_{L}{ }^{*}$ and $C_{M}{ }^{*}$ have been described above, $C_{D}$ is the static drag coefficient at $\alpha_{0}$. Effects of chord-wise aerodynamic admittance $[15,16]$ on the aerodynamic loading are neglected. 


\section{Simulation of measurement errors in the aeroelastic loading}

The "layout" of the stochastic state-space model [15] is derived from the backbone structure of eqns (3)-(5). In contrast with previous investigators ([4-6, 17]), both sources of loading (aeroelastic, AE and buffeting, b) are retained in the stochastic dynamic equations, even though linear superposition of the two effects is still postulated (e.g., $M=M_{A E}+M_{b}$ for the torque in Figure 1). "State augmentation" is used [18] to allow for error propagation. A nonlinear system of Itô-type stochastic differential equations (SDE) [18] is derived from eqns (3)-(5) in terms of a random vector $\mathbf{Z}(s)$ and a dimensionless scalar Wiener process $W(s)$ with time $s$. The stochastic equation [18] is:

$$
d \mathbf{Z}(s)=\mathbf{a}_{N L}(\mathbf{Z}(s)) d s+\sqrt{2 \pi} \mathbf{d} d W(s) .
$$

In eqn (6) $\mathbf{a}_{N L}$ is a nonlinear function of $\mathbf{Z}(\mathrm{s})=\left[\mathbf{Z}_{A E}(\mathrm{~s}), \mathbf{Z}_{T B}(\mathrm{~s}), \Delta\right]^{T}$ with: $\mathbf{Z}_{T B}(s)$ "wind turbulence states", derived from generalized loading $F_{b, k}(s)$ and eqns (45); $\mathbf{Z}_{A E}(\mathrm{~s})$ "aeroelastic and physical states" $[15,19]$, including the generalized coordinates of modes $j$ and $k, \xi_{j}(s), \xi_{k}(s)$, their derivatives with respect to $s, \xi_{j}^{\prime}(s)$, $\xi_{k}^{\prime}(s)$, and the quantities $v_{a e, g, r q, i}$ and $\mu_{a e, g, r q, i}$, described above. The random variable $\Delta$ is independent of time and is used to simulate errors in the aeroelastic loading, for example emerging during the extraction of FDs in wind tunnel.

Equation (6) depends on initial conditions at $s=0$; these are imposed in terms of the joint-probability of $\mathbf{Z}(0)$ at "initial-time", which also includes $\Delta$.

The quantity $\mathbf{d}$ in eqn (6) is a constant vector, employed to model the diffusion term of the SDE, and is related to quantities pertaining to the turbulence-induced loads. These are $\hat{u}(s)=u(s) / U$ and $\hat{w}(s)=w(s) / U$, introduced in Section 2; they are modeled as two independent processes, depending on $W(s)$. For example $\hat{u}(s)$ is written for "Itô-type" SDE as

$$
d \hat{u}(s)=-G_{T B, 1 u} \hat{u}(s) d s+G_{T B, 2 u} d W(s) .
$$

In eqn (7) $G_{T B, 1 u}$ and $G_{T B, 2 u}$ are the parameters of the "turbulence filter" $[1,3]$. A similar model as in eqn (7) is used for $\hat{w}(s)=w(s) / U$, expressed in terms of $G_{T B, 1 w}$ and $G_{T B, 2 w}$. Previous studies $[1,20]$ have demonstrated that the first-order model in eqn (7) is suitable. In fact, the latter parameters can be estimated from the power spectral densities of the wind turbulence at deck level, as described in $[2,20]$.

The effects of aeroelastic modeling errors are indirectly simulated through parameter $\Delta$ in $\mathbf{Z}$. This $\Delta$ is related to the $d_{\tilde{i}, \alpha \alpha}$ coefficient of the IF $\Phi_{\alpha \alpha}$ in eqn (2) (with $i=\tilde{i}=m_{\alpha \alpha}$ ), corresponding to the aeroelastic state $\mu_{a e, k, \alpha \alpha, \tilde{i}}(s)$ of the torsional mode $k$, since this mode is primarily responsible for flutter. The randomization of $d_{\tilde{i}, \alpha \alpha}$ is based on $d_{\tilde{i}, \alpha \alpha}=\bar{d}_{\tilde{i}, \alpha \alpha}+\delta_{\tilde{i}, \alpha \alpha}$, with $\bar{d}_{\tilde{i}, \alpha \alpha}$ being the expected value of the $\tilde{i}$-th term of $\Phi_{\alpha \alpha}$ in eqn (2), while $\Delta=\delta_{\tilde{i}, \alpha \alpha}$ is a zero-mean random parameter. 
The vector $\mathbf{Z}_{A E}(\mathrm{~s})$ in eqn (6) is therefore partitioned as $\mathbf{Z}_{A E}(s)=\left[\mathbf{Z}_{A E}{ }^{\left[\left(n_{A E}-1\right) \times 1\right]}(s), \mu_{a e, k, \alpha \alpha, \tilde{i}}(s)\right]^{T}$ to highlight the contamination error in the last term (mode $k, \Phi_{\alpha \alpha}$ and $i=\tilde{i}=m_{\alpha \alpha}$ ). The IF model requires that

$$
\mu_{a e, k, \alpha \alpha, \tilde{i}}^{\prime}(s)=\left(\bar{d}_{\tilde{i}, \alpha \alpha}+\delta_{\tilde{i}, \alpha \alpha}\right) c_{\tilde{i}, \alpha \alpha} \xi_{k}(s)-\left(\bar{d}_{\tilde{i}, \alpha \alpha}+\delta_{\tilde{i}, \alpha \alpha}\right) \mu_{a e, k, \alpha \alpha, \tilde{i}}(s) .
$$

Other aeroelastic states are unaffected by the random $\delta_{\tilde{i}, \alpha \alpha}$. The error propagation with $d_{\tilde{i}, \alpha \alpha}=\bar{d}_{\tilde{i}, \alpha \alpha}+\delta_{\tilde{i}, \alpha \alpha}$ does not influence mode $j$ because of $\Lambda_{j j, \alpha \alpha}=\Lambda_{j k, \alpha h}=0$ in eqns (3), (6) due to $h_{k}(x)=0$ and $\alpha_{j}(x)=0$.

The dynamics of $\mathbf{Z}_{A E}(s)$ is linear when the error is absent and can be related to a matrix $\mathbf{A}_{A E}$ of dimensions $n_{A E} \times n_{A E}$ in the state-space model, derived from eqn (3). Details may be found in [1]. When $d_{\tilde{i}, \alpha \alpha}=\bar{d}_{\tilde{i}, \alpha \alpha}+\delta_{\tilde{i}, \alpha \alpha}$ is introduced, the first $\left(n_{A E^{-}}-1\right)$ rows of $\mathbf{A}_{A E}$ are still unaffected by eqn (8).

Similarly, the vector-equation related to $\mathbf{Z}_{T B}(s)=[\hat{u}(s), \hat{w}(s)]^{T}$ is linear through an $n_{A E} \times 2$ matrix $\mathbf{A}_{T B}$ :

$$
\mathbf{A}_{T B}^{\left[n_{A E} \times 2\right]}=\left[\begin{array}{cc}
\mathbf{0}^{[2 \times 1]} & \mathbf{0}^{[2 \times 1]} \\
2 Q_{j}\left(\begin{array}{c}
C_{L} \bar{L}_{\psi h_{j}} \\
+C_{M} \bar{L}_{\psi \alpha_{j}}
\end{array}\right) & Q_{j}\left(\begin{array}{c}
\left(C_{D}+C_{L}^{*}\right) \bar{L}_{\psi h_{j}} \\
+C_{M}^{*} \bar{L}_{\psi \alpha_{j}}
\end{array}\right) \\
2 Q_{k}\left(\begin{array}{c}
C_{L} \bar{L}_{\psi h_{k}} \\
+C_{M} \bar{L}_{\psi \alpha_{k}}
\end{array}\right) & Q_{k}\left(\begin{array}{c}
\left(C_{D}+C_{L}^{*}\right) \bar{L}_{\psi h_{k}} \\
+C_{M}^{*} \bar{L}_{\psi \alpha_{k}}
\end{array}\right) \\
\mathbf{0}^{\left[\left(n_{A E}-4\right) \times 1\right]} & \mathbf{0}^{\left[\left(n_{A E}-4\right) \times 1\right]}
\end{array}\right],
$$

with $Q_{j}=0.5 \rho B^{4} \ell / I_{j}$ and $Q_{k}=0.5 \rho B^{4} \ell / I_{k}$. Finally, the nonlinear function of $\mathbf{Z}(s)=\left[\mathbf{Z}_{A E}(\mathrm{~s}), \mathbf{Z}_{T B}(s), \delta_{\tilde{i}, \alpha \alpha}\right]^{T}$ in eqn (6) is rewritten as

$$
\mathbf{a}_{N L}(\mathbf{Z}(s))=\left\{\begin{array}{l}
\mathbf{A}_{A E}^{\left[\left(n_{A E}-1\right) \times n_{A E}\right]} \mathbf{Z}_{A E}(s)+\mathbf{A}_{T B}^{\left[\left(n_{A E}-1\right) \times 2\right]} \mathbf{Z}_{T B}(s) \\
c_{\tilde{i}, \alpha \alpha}\left(\bar{d}_{\tilde{i}, \alpha \alpha}+\delta_{\tilde{i}, \alpha \alpha}\right) \xi_{k}-\left(\bar{d}_{\tilde{i}, \alpha \alpha}+\delta_{\tilde{i}, \alpha \alpha}\right) \mu_{a e, k, \alpha \alpha, \tilde{i}} \\
\mathbf{T}_{u w} \mathbf{Z}_{T B}(s) \\
0
\end{array}\right\} .
$$

Equation (10) is combined with $\mathbf{d}=\left[\mathbf{0}^{\left[1 \times n_{A E}\right]}, G_{T B, 2 u}, G_{T B, 2 w}, 0\right]^{T}$ and the matrix

$$
\mathbf{T}_{u w}=\left[\begin{array}{cc}
-G_{T B, 1 u} & 0 \\
0 & -G_{T B, 1 w}
\end{array}\right] .
$$

In previous eqns (9)-(11) the superscripts, included within brackets, denote the dimensions of each sub-matrix or vector. 


\section{4 "Direct Euler Monte-Carlo" estimation of statistical moments vs. assessment of moment Lyapunov exponents}

The concept of Mean-Square Stability (MSS), based on the second-moments properties of $\mathrm{E}\left[\mathbf{Z}(s) \mathbf{Z}(s)^{T}\right]$, has often been utilized (e.g. [4-6, 17]) for determining the role of noise on the critical flutter velocity $\left(U_{c r}\right)$ as a function of the initial distribution of $\mathbf{Z}(s=0)$. Recently, it has been shown [9] that an MSSbased estimation may not be conservative in a nonlinear SDE system (as in eqn (6)), and that a smaller stochastic stability domain, i.e., a smaller $U_{c r}$, may be possible if higher statistical moments are considered.

The latter observation has been used to evaluate the flutter velocity in the presence of IF errors. The evaluation of $U_{c r}$ is based on the definition of " $p$-th moment stochastic stability" [9] of the time-varying process $\mathbf{Z}(s)$ in eqn (6) with $p=2$ and $p=3$. First-moment stability $(p=1)$ has not been analyzed since $U_{c r}$ coincides with deterministic flutter, independently of $\mathbf{a}_{N L}[1]$.

First, a "Direct Euler-Monte Carlo" algorithm (DEMC) is employed to solve eqn (6) numerically, as closed-form solution of eqns (6) ([4-6,17]) is impractical when the number of states is large. The DEMC algorithm is based on the Euler time-marching scheme [10, 18] for integrating the SDE, and on Monte Carlo simulation for assessing statistical moments. It requires the generation of $t^{\prime}=0, . ., N_{S}$ samples of unit-variance Gaussian increments $\left(\Delta W_{j^{\prime}}^{\left(t^{\prime}\right)}\right)$ of the Wiener process $W(s)$. Such increments are evaluated between $s_{j^{\prime}-1}$ and $s_{j^{\prime}}$ time instants, i.e., for step $\Delta s_{j}{ }^{\left(t^{\prime}\right)}=s_{j}{ }^{\left(t^{\prime}\right)}-s_{j-1}{ }^{\left(t^{\prime}\right)}$ of index $j^{\prime}$. As a result, eqn (6) can be transformed into $N_{S}$ deterministic iterative problems with respect to index $t$ ' as

$$
\mathbf{Z}^{(t)}\left(s_{j^{\prime}}\right)=\mathbf{Z}^{(t)}\left(s_{j^{\prime}-1}\right)+\mathbf{a}_{N L}\left(\left[\mathbf{Z}_{A E}{ }^{(t)}\left(s_{j^{\prime}-1}\right), \mathbf{Z}_{T B}{ }^{\left(t^{t}\right)}\left(s_{j^{\prime}-1}\right), \delta_{i}\right]^{T}\right) \Delta s_{j^{\prime}}^{\left(t^{\prime}\right)}+\sqrt{2 \pi} \mathbf{d} \Delta W_{j^{\prime}}^{(t)}
$$

In eqns (12) the increments of $W(s), \Delta W_{j^{\prime}}^{\left(t^{\prime}\right)}$, are mutually independent. A Monte-Carlo-based sample of the random vector at initial time, $\mathbf{Z}^{\left(t^{\prime}\right)}\left(s_{0}=0\right)$, is also generated. Since eqn (12) converges to the solution of eqn (6) as $N_{S} \rightarrow \infty$ [10] in "weak form", the DEMC algorithm is employed to estimate the $p$-th moments of the modal coordinates $\left(\mathrm{E}\left[\xi_{g}(s)^{2}\right]\right.$ or $\mathrm{E}\left[\xi_{g}(s)^{3}\right]$ with $\left.g=j, k\right)$ and "modal velocities" $\left(\mathrm{E}\left[\xi_{g}^{\prime}(s)^{2}\right]\right.$ or $\left.\mathrm{E}\left[\xi_{g}^{\prime}(s)^{3}\right]\right)$. Preliminary numerical tests [1] have indicated that, since the latter quantities are mainly responsible for flutter, the analysis of other states is redundant. In contrast with most literature examples (e.g. [9]) the temporal evolution of $\mathrm{E}\left[\xi_{g}(s)^{3}\right]$ is used herein, instead of $\mathrm{E}\left[\left|\xi_{g}(s)\right|^{3}\right]$.

In parallel with the DEMC algorithm, the $p$-th Moment Lyapunov Exponent (MLE) $[9,21]$ of the state vector $\mathbf{Z}$ is calculated. The assessment of the $p$-th MLE [9] is based on a suitable norm of $\mathbf{Z}$. The MLE is related to the slowest oscillation decay rate in an SDE system and is equivalent to the largest eigenvalue of a deterministic system. A negative MLE indicates $p$-th moment stability [9]. In this study, the assessment of the $p$-th MLE has been restricted to the sub- 
vector $\boldsymbol{\Xi}(s)=\left[\xi_{j}(s), \xi_{k}(s), \xi_{j}^{\prime}(s), \xi_{k}{ }_{k}(s)\right]^{T}$, mainly responsible for flutter, and for comparison with the DEMC algorithm. The $p$-th MLE of $\Xi(s)$ is [9]

$$
\Lambda_{\Xi}(p)=\lim _{s \rightarrow \infty} \frac{1}{s} \log \left(\mathrm{E}\left[\|\Xi(s)\|^{p}\right]\right) .
$$

The term $\|\boldsymbol{\Xi}(s)\|^{p}=\left(\boldsymbol{\Xi}(s)^{T} \boldsymbol{\Xi}(s)\right)^{p / 2}$ is derived from the Euclidean norm of $\boldsymbol{\Xi}(s)$ with $p=2,3$. Practically, eqn (13) is estimated as $\Lambda_{\Xi}(p) \approx 1 / s \log \left(\mathrm{E}\left[\|\boldsymbol{\Xi}(s)\|^{p}\right]\right)$ for a large $s$ by Euler - Monte Carlo integration in eqns (13). Even though more refined procedures for estimating $\Lambda_{\Xi}(p)$ are available [9], this simple approach is still acceptable. Errors, numerical convergence and approximation of the exact solution are known issues of both DEMC algorithm and an approximated MLE [9]. Both aspects have been carefully addressed by controlling the time step $\Delta s_{j^{\prime}}{ }^{\left({ }^{\prime}\right)}$ and by extending each Monte Carlo sample of eqns (12) over a long time.

\section{Simulation results}

A simplified long-span bridge model, designated as "B1", is used in this preliminary analysis. The structural properties of B1 are derived from the 1992 design of the Messina Strait Bridge Italy, [22]. This is a suspension bridge with central span length $\ell_{c s}=3300 \mathrm{~m}$, deck width $B=60 \mathrm{~m}$, mass per unit length $m_{0}=5.4 \times 10^{4} \mathrm{~kg} / \mathrm{m}$ and mass moment of inertia $I_{0}=28.0 \times 10^{6} \mathrm{~kg} \times \mathrm{m}^{2} / \mathrm{m}$. Flexural $(j)$ and torsional $(k)$ modes are skew-symmetric with frequencies 0.06 $\mathrm{Hz}, 0.08 \mathrm{~Hz}$. Mode shapes are $h_{j, \mathrm{~B} 1}(x)=\sin \left(2 \pi x / \ell_{c s}\right), \alpha_{k, \mathrm{~B} 1}(x)=\sin \left(2 \pi x / \ell_{c s}\right)$.

The aerodynamic and aeroelastic properties of the "flat plate" [8] are employed to approximately simulate the behavior of a streamlined closed-box girder. Static coefficients at $\alpha_{0}=0$ are: $C_{L}=C_{M}=0, C^{*}{ }_{L}=2 \pi$ (positive upward) and $C^{*}{ }_{M}=\pi / 2$. The "error-free" parameters (as in eqn 2) are the same for both lift and torque loading due to $\Phi_{h \alpha}(s)=\Phi_{h h}(s)=\Phi_{\alpha h}(s)=\Phi_{\alpha \alpha}(s)$ with model order $m_{h \alpha}=m_{h h}=m_{\alpha h}=m_{\alpha \alpha}=2$. In the case of $\Phi_{\alpha \alpha}(s)$ with $s=t U / B$, these coincide with the R.T. Jones' parameters [23] $c_{1, \alpha \alpha}=0.165, d_{1, \alpha \alpha}=0.09, c_{2, \alpha \alpha}=0.335$, and the average $\bar{d}_{2, \alpha \alpha}=0.60$. The deterministic flutter velocity $\left(\delta_{2, \alpha \alpha}=0\right)$ is $20.1 \mathrm{~m} / \mathrm{s}$ for B1 $[1,2]$.

The Kaimal and the Lumley-Panowsky spectra are chosen to describe the turbulences $\hat{u}$ and $\hat{w}$. For $15 \mathrm{~m} / \mathrm{s}<U<30 \mathrm{~m} / \mathrm{s}$ at deck elevation (eqn 7) it can be found $[1,2]: G_{T B, 1 u}=0.186, G_{T B, 2 u}=0.066, G_{T B, 1 w}=1.489, G_{T B, 2 w}=0.104$. The dimensionless span-wise correlation lengths in eqns (4-5) are $\bar{L}_{\psi_{h j}}=\bar{L}_{\psi \alpha k}=0.11$.

Stochastic stability is numerically investigated by DEMC and MLE under the following assumptions: modal responses at time $s=0$ (initial amplitudes $\xi_{j 0}$ and $\left.\xi_{k 0}\right)$ are two independent jointly-Gaussian processes with zero mean and dimensionless variances $\mathrm{E}\left[\xi_{j 0}{ }^{2}\right]=\mathrm{E}\left[\xi_{k 0}{ }^{2}\right]$, variable between 0.01 (small initial perturbation, compatible with buffeting vibration regimes for a long-span bridge) and 1.00 (large perturbation, possibly unrealistic). The IF measurement error $\delta_{2, \alpha \alpha}$ is a Gaussian variable, independent of other $\mathbf{Z}$ states and of time $s$, with zero mean and Standard Deviation $\operatorname{SD}\left(\delta_{2, \alpha \alpha}\right)=0.22$. Inspection has revealed that for 
B1, the step $\Delta s_{j^{\prime}}{ }^{(t)}$ in eqns (12) must coincide with a time duration $\Delta t$ at least $10^{-3}$ times smaller than the lowest period of interest, and that $N_{S} \geq 150$ must be used for accurate assessment of $\mathrm{E}\left[\xi_{j}(s)^{p}\right]$ and $\mathrm{E}\left[\xi_{k}(s)^{p}\right]$.

Figure 2 shows a typical example of the numerical results, based on the DEMC algorithm. Second and third moments of the torsional mode $k$ at $U=18.5$ $\mathrm{m} / \mathrm{s}$ are depicted as a function of dimensionless time $s$ with initial variances $\mathrm{E}\left[\xi_{j 0}{ }^{2}\right]=\mathrm{E}\left[\xi_{k 0}{ }^{2}\right]=0.10$ at $s=0$ for both modes. Dimensionless modal amplitudes and velocities are depicted. It is evident from Figure 2 that this velocity is unstable for B1 if the 3-rd moment stability criterion is utilized (modal velocity diverges for $s>50$ ), whereas $\mathrm{E}\left[\xi_{k}^{2}(s)\right]$ ) of $\mathrm{B} 1$ is still stable (bounded vibration).
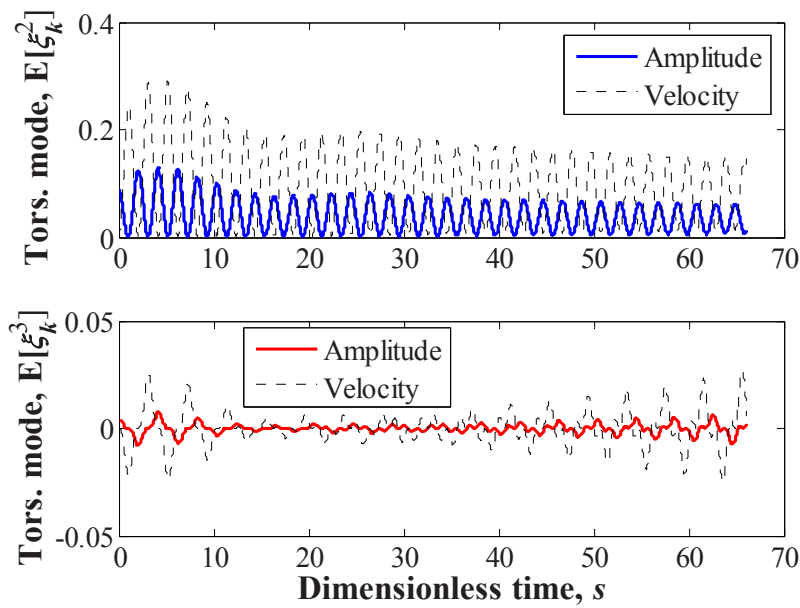

Figure 2: DEMC-based estimation of $2^{\text {nd }}-\left(E\left[\xi_{k}^{2}(s)\right]\right)$ and $3^{\text {rd }}$-moment $\left(\mathrm{E}\left[\xi_{k}^{3}(s)\right]\right)$ time histories for mode $k$ of B1 at $U=18.5 \mathrm{~m} / \mathrm{s}$. Zeromean IF error with $\operatorname{SD}\left(\delta_{2, \alpha \alpha}\right)=0.22$ and initial variance $\mathrm{E}\left[\xi_{k 0}{ }^{2}\right]=0.10$.

Figure 3 shows an example of $2^{\text {nd }}$ and $3^{\text {rd }}$ MLE estimation for B1 at $U=18.5$ $\mathrm{m} / \mathrm{s}$, based on the time history of $\Lambda_{\Xi}(p) \approx 1 / s \log \left(\mathrm{E}\left[\|\boldsymbol{\Xi}(s)\|^{p}\right]\right)$, zero-mean IF error with $\operatorname{SD}\left(\delta_{2, \alpha \alpha}\right)=0.22$ and initial variances $\mathrm{E}\left[\xi_{j 0}{ }^{2}\right]=\mathrm{E}\left[\xi_{k 0}{ }^{2}\right]=0.10$. This figure is equivalent to Figure 2 since $U$, initial conditions imposed on $\xi_{j 0}$ and $\xi_{k 0}$ and statistical properties of $\delta_{2, \alpha \alpha}$ are the same. It is utilized for comparison purposed between DEMC and MLE methods.

In Figure 3 a transition from negative to positive values can be seen for both $\Lambda_{\Xi}(2)$ and $\Lambda_{\Xi}(3)$ at a very large $s>150$; this fact suggests that, based on MLE estimation (and in contrast with Figure 2), flutter has already occurred for B1 at $U=18.5 \mathrm{~m} / \mathrm{s}$, independently of the moment stability criterion.

Finally, Figure 4 is a summary of the simulation results for B1. In Figure 4, the flutter velocity $\left(U_{c r}\right)$ of $\mathrm{B} 1$ is shown as a function of $\mathrm{E}\left[\xi_{j 0}{ }^{2}\right]=\mathrm{E}\left[\xi_{k 0}{ }^{2}\right]$. The comparison of results is based on $2^{\text {nd }}(p=2)$ and $3^{\text {rd }}(p=3)$ moment stability criteria, calculated by both DEMC and MLE methods. Resolution in Figure 4 is $\pm 0.5 \mathrm{~m} / \mathrm{s}$. As a general trend, a reduction of $U_{c r}$ is observed as a function of initial amplitude vibration variances. 


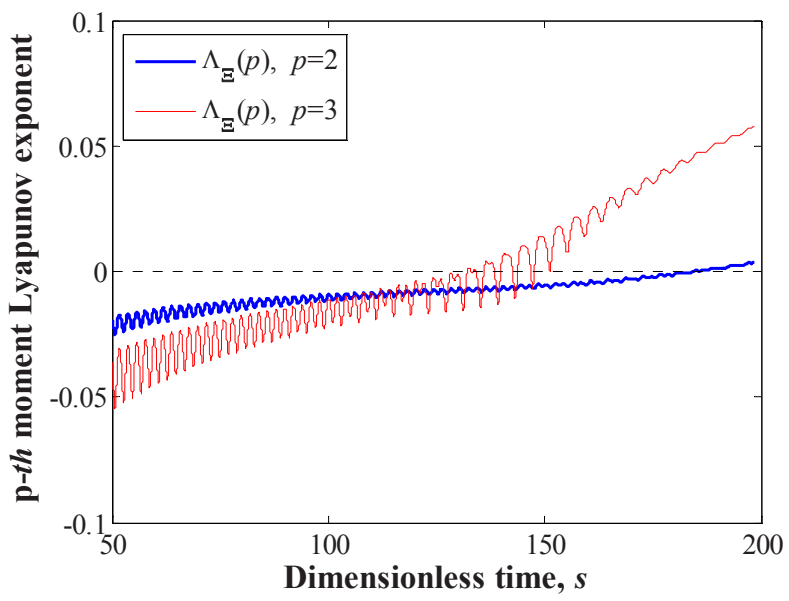

Figure 3: Assessment of $2^{\text {nd }}$ and $3^{\text {rd }}$ MLE for B1 at $U=18.5 \mathrm{~m} / \mathrm{s}$, based on the time history of $\Lambda_{\Xi}(p) \approx 1 / s \log \left(\mathrm{E}\left[\|\Xi(s)\|^{p}\right]\right)$. Zero-mean IF error with $\operatorname{SD}\left(\delta_{2, \alpha \alpha}\right)=0.22$ and initial variances $\mathrm{E}\left[\xi_{j 0}{ }^{2}\right]=\mathrm{E}\left[\xi_{k 0}{ }^{2}\right]=0.10$.

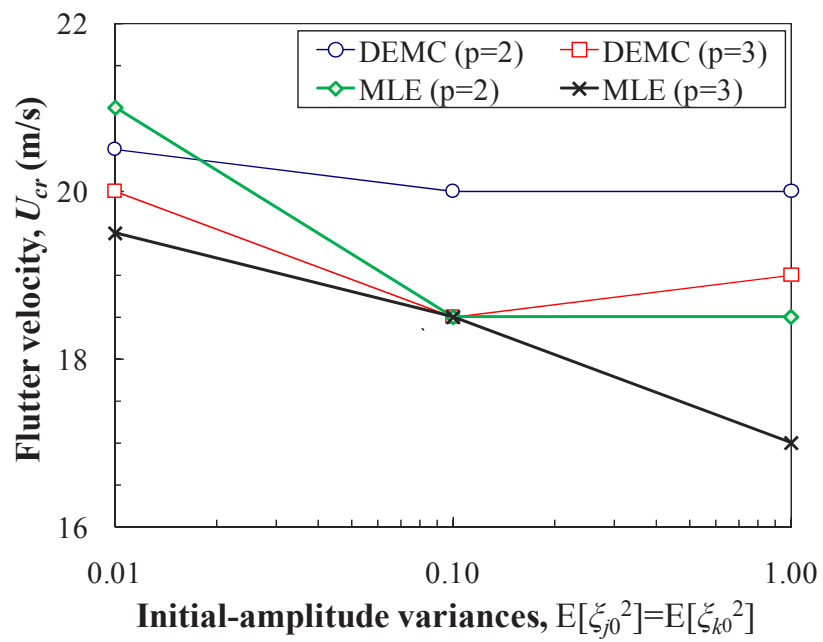

Figure 4: Flutter velocity of B1 vs. initial-amplitude variances $\left(\mathrm{E}\left[\xi_{j 0}^{2}\right]=\mathrm{E}\left[\xi_{k 0}^{2}\right]\right) ; p$-th moment stability calculated by DEMC and MLE. Zero-mean IF error with $\operatorname{SD}\left(\delta_{2, \alpha \alpha}\right)=0.22$.

\section{Discussion and concluding remarks}

Coupled-mode Flutter stability of a long-span bridge, influenced by the randomness in the aeroelastic bridge properties, is investigated in this study. An analytically-derived and numerically-implement model, based on stochastic differential equations, has been utilized. Stochastic stability threshold criteria 
have been established in terms of mean squares and third moments. A 3300m simulated bridge model has been employed as a preliminary test case. A decrement of critical velocity has been noticed if the variances of initial vibration amplitude are increased. The latter quantity may be interpreted as being related to an initial deck oscillation at incipient flutter due to wind turbulence The observed decrement of critical velocity is not negligible, between $10 \%$ and $20 \%$, depending on the stability criterion which is employed. The study has also confirmed the feasibility of using Moment Lyapunov Exponents for random flutter estimation. More investigation is still needed, since some differences have been noticed among various stability criteria.

\section{Acknowledgements}

This research was supported in part by the National Science Foundation of the United States (NSF), Award No. 0600575. The support of NSF for performancebased dynamic-response assessment of slender structures is also acknowledged (CAREER Award No. 0844977). Any opinions, observations and conclusions are those of the writer and do not necessarily reflect the views of the NSF.

\section{References}

[1] Caracoglia, L., Simulation of linear and non-linear propagation effects of a random turbulence field on bridge flutter instability, Journal of Wind Engineering and Industrial Aerodynamics (in review), 2009.

[2] Caracoglia, L., Some implications of the effects of turbulence on bridge flutter. Proc. of the Fourth International Conference on 'Advances in Wind and Structures (AWAS'08)', Jeju, South Korea, pp. 1667-1675, 2008.

[3] Bartoli, G., Borri, C. \& Gusella, V., On the influence of wind turbulence on bridge deck flutter. Aspects in Modern Computational Structural Analysis, eds. Meskouris \& Vittek, Balkema: Rotterdam, NL, pp. 3-17, 1997.

[4] Bucher, C.G. \& Lin, Y.K., Stochastic stability of bridges considering coupled modes, Journal of Engineering Mechanics, ASCE, 114(12), pp. 2055-2071, 1988.

[5] Tsiatas, G. \& Sarkar, P.P., Motion stability of long-span bridges under gusty winds, Journal of Engineering Mechanics, ASCE, 114(2), pp. 257276, 1988.

[6] Sepe, V. \& Vasta, M., Turbulence effects on the aeroelastic stability of long-span bridges. Proc. of the Mini-Symposium on "Large Bridge Aerodynamics and Aeroelastic Phenomena", Sixth European Conference on Structural Dynamics (EuroDyn 2005), Paris, France, pp. 421-426, 2005.

[7] Pospíšil, S., Náprstek, J. \& Hracov, S., Stability domains in flow-structure interaction and influence of random noises, Journal of Wind Engineering and Industrial Aerodynamics, 94(11), pp. 883-893, 2006.

[8] Scanlan, R.H., Béliveau, J.G. \& Budlong, K.S., Indicial aerodynamic functions for bridge decks, Journal of Engineering Mechanics, ASCE, 100(EM4), pp. 657-673, 1974. 
[9] Xie, W.-C., Dynamic Stability of Structures, Cambridge University Press: New York, NY, USA, 2006.

[10] Kloeden, P.E. \& Platen, E., Numerical Solution of Stochastic Differential Equations through Computer Experiments, Springer-Verlag: BerlinHeidelberg, Germany, 1992.

[11] Jones, N.P. \& Scanlan, R.H., Theory and full-bridge modeling of wind response of cable-supported bridges, Journal of Bridge Engineering, ASCE, 6(6), pp. 365-375, 2001.

[12] Katsuchi, H., Jones, N.P. \& Scanlan, R.H., Multimode coupled buffeting and flutter analysis of the Akashi-Kaikyo Bridge, Journal of Structural Engineering, ASCE, 125(1), pp. 60-70, 1999.

[13] Scanlan, R.H. \& Tomko, J.J., Airfoil and bridge deck flutter derivatives, Journal of Engineering Mechanics, ASCE, 97(EM6), pp. 1717-1737, 1971.

[14] Caracoglia, L. \& Jones, N.P., Time domain vs. frequency domain characterization of aeroelastic forces for bridge deck sections, Journal of Wind Engineering and Industrial Aerodynamics, 91(3), pp. 371-402, 2003.

[15] Chen, X., Matsumoto, M. \& Kareem, A., Time domain flutter and buffeting response analysis of bridges, Journal of Engineering Mechanics, 126(1), pp. 7-16, 2000.

[16] Scanlan, R.H. \& Jones, N.P., A form of aerodynamic admittance for use in bridge aeroelastic analysis, Journal of Fluids and Structures, 13(7-8), pp. 1017-1027, 1999.

[17] Bartoli, G. \& Spinelli, P., The stochastic differential calculus for the determination of structural response under wind, Journal of Wind Engineering and Industrial Aerodynamics, 48(2-3), pp. 175-188, 1993.

[18] Grigoriu, M., Stochastic Calculus. Applications in Science and Engineering, Birkhäuser: Boston, MA, USA, 2002.

[19] Lin, Y.K. \& Ariaratnam, S.T., Stability of bridge motion in turbulent winds, Journal of Structural Mechanics, 8(1), pp. 1-15, 1980.

[20] Caracoglia, L., Influence of uncertainty in selected aerodynamic and structural parameters on the buffeting response of long-span bridges, Journal of Wind Engineering and Industrial Aerodynamics, 96(3), pp. 327344, 2008.

[21] Ariaratnam, S.T. \& Xie, W.-C., Lyapunov exponents and stochastic stability of two-dimensional parametrically excited random systems, Journal of Applied Mechanics, ASME, 60(3), pp. 677-682, 1993.

[22] Sepe, V., Caracoglia, L. \& D’Asdia, P., Aeroelastic instability of long-span bridges: contributions to the analysis in frequency and time domains, Wind and Structures, 3(1), pp. 41-58, 2000.

[23] Jones, R.T., The unsteady lift of a finite wing, Technical Note 682, NACA, 1939. 